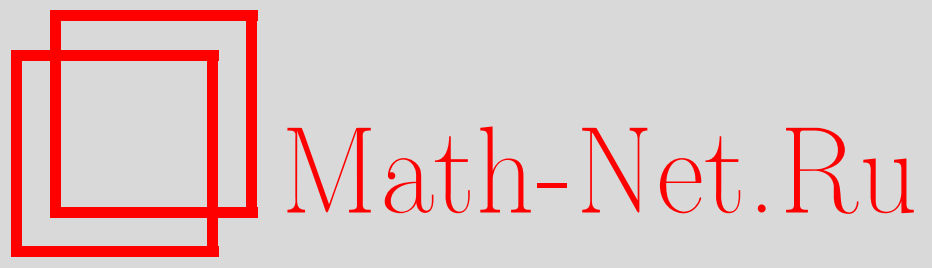

А. Н. Старцев, Об одном подходе к оценке параметров двумерного процесса линейной диффузии в нестационарном случае, Теория вероятн. и ее примен., 2014, том 59, выпуск 2, 386-391

DOI: https://doi.org/10.4213/tvp4571

Использование Общероссийского математического портала Math-Net.Ru подразумевает, что вы прочитали и согласны с пользовательским соглашением http://www . mathnet.ru/rus/agreement

Параметры загрузки:

IP: 54.80 .73 .141

26 апреля 2023 г., 10:09:30

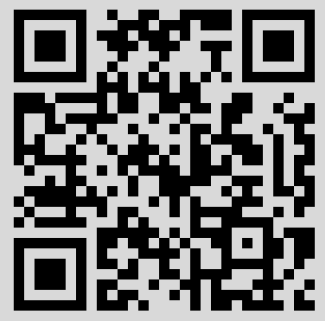


3. Peszat S., Zabczyk J. Stochastic partial differential equations with Lévy noise: an evolution equation approach. Cambridge: Cambridge Univ. Press, 2007, 419 p.

4. Duncan T.E., Pasik-Duncan B., Maslowski B. Fractional Brownian motion and stochastic equations in Hilbert spaces. - Stoch. Dyn., 2002, v. 2, № 2, p. 225-250.

5. Brzeźniak Z., van Neerven J. Stochastic convolution in separable Banach space and the stochastic linear Cauchy problem. - Studia Math., 2000, v. 143, № 1, p. 43-74.

6. van Neerven J.M.A.M., Weis L. Stochastic integration of functions with values in a Banach space. - Studia Math., 2005, v. 166, № 2, p. 131-170.

7. van Neerven J., Veraar M., Weis L. Stochastic evolution equations in UMD Banach spaces. - J. Funct. Anal., 2008, v. 255, № 4, p. 940-993.

8. Dettweiler J., Weis L., van Neerven J. Space-time regularity of solutions of the parabolic stochastic Cauchy problem. - Stoch. Anal. Appl., 2006, v. 24, № 4, p. 843869.

9. Mémin J., Mishura Yu., Valkeila E. Inequalities for the moments of Wiener integrals with respect to a fractional Brownian motion. - Statist. Probab. Lett., 2001, v. 51, № 2, p. 197-206.

10. Samorodnitsky G., Taqqu M.S. Stable Non-Gaussian Random Processes. Boca Raton: Chapman \& Hall, 1994, 632 p.

11. Kwapień S., Woycziński W. A. Random Series and Stochastic Integrals: Single and Multiple. Boston: Birkhäuser, 1992, 360 p.

12. Радченко В. Н. Интегралы по общим случайным мерам. Киев: Ин-т математики НАН Украины, 1999, 196 с. (Труды Института математики НАН Украины, т. 27.)

13. Радченко В. Н. Интегралы по случайным мерам и случайные линейные функционалы. - Теория вероятн. и ее примен., 1991, т. 36, в. 3, с. 594-596.

14. Радченко В.Н. Выборочные функции случайных мер и пространства Бесова. Теория вероятн. и ее примен., 2009, т. 54, в. 1, с. 158-166.

15. Drewnowski L. Topological rings of sets, continuous set functions, integration. III. Bull. Acad. Pol. Sci., 1972, v. 20, p. 439-445.

16. Вахания Н.Н., Тариеладзе В.И., Чобанян С. А. Вероятностные распределения в банаховых пространствах. М.: Наука, 1985, 368 с.

17. Radchenko V. M. Besov regularity of stochastic measures. - Statist. Probab. Lett., 2007 , v. 77 , № 8, p. 822-825.

18. Drewnowski $L$. Boundness of vector measures with values in the spaces $L_{0}$ of Bochner measurable functions. - Proc. Amer. Math. Soc., 1984, v. 91, p. 581-588.

19. Pazy A. Semigroups of Linear Operators and Applications to Partial Differential Equations. New York: Springer-Verlag, 1983, 280 p.

20. Kamont A. A discrete characterization of Besov spaces. - Approx. Theory Appl. (N.S.), 1997, v. 13, № 2, p. 63-77.

Поступила в редакцию 24.V.2013

Исправленный вариант 22.X.2013

(c) 2014 г.

СТАРЦЕВ А.Н.*

\title{
ОБ ОДНОМ ПОДХОДЕ К ОЦЕНКЕ ПАРАМЕТРОВ ДВУМЕРНОГО ПРОЦЕССА ЛИНЕЙНОЙ ДИФФУЗИИ В НЕСТАЦИОНАРНОМ СЛУЧАЕ
}

\begin{abstract}
Рассмотрены отличные от оценок максимального правдоподобия оценки параметров двумерного марковского гауссовского нестационарного процесса, заданного системой линейных стохастических уравнений с двумя параметрами. Доказана их асимптотическая нормальность и
\end{abstract}

* Институт математики им. В. И. Романовского АН РУз, Ташкент, Узбекистан; e-mail: anstartsev@gmail.com 
асимптотическая независимость, что позволяет строить доверительные интервалы одновременно для обоих параметров.

Ключевые слова и фразы: двумерный гауссовский процесс, линейная диффузия, нестационарный случай, оценка параметров, асимптотические свойства.

1. Введение. Пусть на интервале $[0, T]$ наблюдается двумерный гауссовский процесс $\xi(t)=\left(\xi_{1}(t), \xi_{2}(t)\right)$, удовлетворяющий следующей системе стохастических дифференциальных уравнений:

$$
\begin{aligned}
& d \xi_{1}(t)=-\lambda \xi_{1}(t) d t-\omega \xi_{2}(t) d t+d w_{1}(t), \\
& d \xi_{2}(t)=-\lambda \xi_{2}(t) d t+\omega \xi_{1}(t) d t+d w_{2}(t),
\end{aligned}
$$

где $w(t)=\left(w_{1}(t), w_{2}(t)\right)$ - двумерный винеровский процесс с независимыми компонентами. Предполагается, что $\mathbf{E} \xi_{1}(t)=\mathbf{E} \xi_{2}(t)=0$, а $\xi(0)$ не зависят от $w(t)$, причем $\xi_{1}(0)$ и $\xi_{2}(0)-$ независимые случайные величины с $\mathbf{E} \xi_{1}^{2}(0)=\mathbf{E} \xi_{2}^{2}(0)=\sigma^{2}$, $0 \leqslant \sigma^{2}<\infty$.

Эта система уравнений была предложена А.Н. Колмогоровым в 1960 г. [1] (см. также [2]) как модель, описывающая колебания мгновенной оси вращения Земли относительно малой оси земного эллипсоида после устранения годовой периодический компоненты (так называемые чендлеровские колебания). В этой же работе в стационарном случае были предложены оценки максимального правдоподобия для параметров $\omega$ и $\lambda$, которые имеют вид

$$
\begin{aligned}
& \widehat{\omega}=\frac{1}{\chi_{2}(T)}\left[\int_{0}^{T} \xi_{1}(t) d \xi_{2}(t)-\int_{0}^{T} \xi_{2}(t) d \xi_{1}(t)\right], \\
& \widehat{\lambda}=\frac{1}{2 \chi_{2}(T)}\left[\sqrt{\left(\chi_{1}(T)-T\right)^{2}+4 \chi_{2}(T)}-\left(\chi_{1}(T)-T\right)\right],
\end{aligned}
$$

где $\chi_{1}(T)=\left(|\xi(0)|^{2}+|\xi(T)|^{2}\right) / 2, \chi_{2}(T)=\int_{0}^{T}|\xi(t)|^{2} d t$.

Там же отмечалось, что $(\widehat{\omega}-\omega) \sqrt{\chi_{2}(T)}$ имеет стандартное нормальное распределение. Строгое обоснование этого факта можно найти в монографии [3] (см. также [2]). Что касается оценки $\widehat{\lambda}$, то в работе [1] были найдены некоторые частные аппроксимации для ее распределения. В [2] был найден явный вид характеристической функции достаточной статистики $\left(\chi_{1}(T), \chi_{2}(T)\right)$, который, однако, имеет довольно сложный вид и зависит от неизвестного параметра $\lambda$. В силу этого распределение было табулировано не только по значением квантилей, но и по значениям параметра $\lambda$.

В данной работе предлагается другой подход к получению оценок, основанный на построении уравнений оценивания, исходя из (1) и (2). Например, если умножить уравнение $(1)$ на $\xi_{2}(t)$, а уравнение $(2)$ на $\xi_{1}(t)$ и проинтегрировать их по $t$ от 0 до $T$, то получим систему

$$
\begin{aligned}
& \int_{0}^{T} \xi_{2}(t) d \xi_{1}(t)=-\lambda \int_{0}^{T} \xi_{1}(t) \xi_{2}(t) d t-\omega \int_{0}^{T} \xi_{2}^{2}(t) d t+\int_{0}^{T} \xi_{2}(t) d w_{1}(t), \\
& \int_{0}^{T} \xi_{1}(t) d \xi_{2}(t)=-\lambda \int_{0}^{T} \xi_{1}(t) \xi_{2}(t) d t+\omega \int_{0}^{T} \xi_{1}^{2}(t) d t+\int_{0}^{T} \xi_{1}(t) d w_{2}(t) .
\end{aligned}
$$

Решая эту систему относительно $\omega$, без учета последних членов, получим оценку (3). Однако оценка для $\lambda$ при этом оказывается существенно более сложной. $\mathrm{C}$ другой стороны, можно получить аналогичную систему, удобную для построения 
оценки параметра $\lambda$ :

$$
\begin{aligned}
& \int_{0}^{T} \xi_{1}(t) d \xi_{1}(t)=-\lambda \int_{0}^{T} \xi_{1}^{2}(t) d t-\omega \int_{0}^{T} \xi_{1}(t) \xi_{2}(t) d t+\int_{0}^{T} \xi_{1}(t) d w_{1}(t), \\
& \int_{0}^{T} \xi_{2}(t) d \xi_{2}(t)=-\lambda \int_{0}^{T} \xi_{2}^{2}(t) d t+\omega \int_{0}^{T} \xi_{1}(t) \xi_{2}(t) d t+\int_{0}^{T} \xi_{2}(t) d w_{2}(t) .
\end{aligned}
$$

Исключая $\omega$, получаем оценку

$$
\widetilde{\lambda}=-\frac{1}{\chi_{2}(T)}\left[\int_{0}^{T} \xi_{1} d \xi_{1}+\int_{0}^{T} \xi_{2} d \xi_{2}\right]=-\frac{|\xi(T)|^{2}-|\xi(0)|^{2}-2 T}{2 \chi_{2}(T)} .
$$

Несмотря на то что полученная оценка имеет более простую структуру в сравнении с оценкой максимального правдоподобия $\widehat{\lambda}$, ее предельное распределение также будет сложным и зависящим от неизвестного параметра, что легко следует из результатов М. Арато [2] относительно оценки $\widehat{\lambda}$.

Ранее аналогичный подход применялся автором в одномерном случае: в [4] для процесса авторегрессии и в [5] для процесса линейной диффузии. Истоки этого метода восходят к работе [6], посвященной оцениванию параметров в регрессионных моделях.

2. Построение оценок и их асимптотические свойства. Нашей основной целью является построение других более простых по структуре оценок, хорошо действующих в нестационарном случае и имеющих простое предельное распределение. и $(2)$ :

Построим теперь уравнения оценивания простым интегрированием уравнений (1)

$$
\begin{aligned}
& \xi_{1}(T)-\xi_{1}(0)=-\lambda \int_{0}^{T} \xi_{1}(t) d t-\omega \int_{0}^{T} \xi_{2}(t) d t+w_{1}(T), \\
& \xi_{2}(T)-\xi_{2}(0)=-\lambda \int_{0}^{T} \xi_{2}(t) d t+\omega \int_{0}^{T} \xi_{1}(t) d t+w_{2}(T) .
\end{aligned}
$$

Обозначая $\xi_{j}(T)-\xi_{j}(0)=\xi_{j}^{0}(T), I_{j}(T)=\int_{0}^{T} \xi_{j}(t) d t, j=1,2$, получим следующую систему (без учета последних членов $w_{1}(T)$ и $w_{2}(T)$ ):

$$
\xi_{1}^{0}(T)=-\lambda I_{1}(T)-\omega I_{2}(T), \quad \xi_{2}^{0}(T)=-\lambda I_{2}(T)+\omega I_{1}(T) .
$$

Решая эту систему относительно $\lambda$ и $\omega$, находим

$$
\lambda^{*}=-\frac{\xi_{1}^{0}(T) I_{1}(T)+\xi_{2}^{0}(T) I_{2}(T)}{I(T)}, \quad \omega^{*}=\frac{\xi_{2}^{0}(T) I_{1}(T)-\xi_{1}^{0}(T) I_{2}(T)}{I(T)},
$$

где $I(T)=I_{1}^{2}(T)+I_{2}^{2}(T)$.

Сформулируем основной результат.

Теорема 1. В наших условиях для всех $\lambda<0 u-\infty<\omega<\infty$ величинь $\left(\lambda^{*}-\lambda\right)(I(T) / T)^{1 / 2}$ u $\left(\omega^{*}-\omega\right)(I(T) / T)^{1 / 2}$ при $T \rightarrow \infty$ являются асимптотически независимьми и имеют в качестве предельного стандартное нормальное распределение.

Д о к а з а т е л ь с т в о. Из (5) легко получить и отклонения построенных оценок от оцениваемых параметров:

$$
\lambda^{*}-\lambda=-\frac{w_{1}(T) I_{1}(T)+w_{2}(T) I_{2}(T)}{I(T)}, \quad \omega^{*}-\omega=\frac{w_{2}(T) I_{1}(T)-w_{1}(T) I_{2}(T)}{I(T)},
$$

которые и служат основой для изучения предельного поведения. В частности, из (6) имеем

$$
\left(\frac{I(T)}{T}\right)^{1 / 2}\left(\lambda^{*}-\lambda\right)=-\left[w_{1}^{*} \frac{\eta(T)}{\sqrt{1+\eta^{2}(T)}}+w_{2}^{*} \frac{1}{\sqrt{1+\eta^{2}(T)}}\right],
$$




$$
\left(\frac{I(T)}{T}\right)^{1 / 2}\left(\omega^{*}-\omega\right)=w_{2}^{*} \frac{\eta(T)}{\sqrt{1+\eta^{2}(T)}}-w_{1}^{*} \frac{1}{\sqrt{1+\eta^{2}(T)}}
$$

где $w_{1}^{*}=w_{1}(T) / \sqrt{T}$ и $w_{2}^{*}=w_{2}(T) / \sqrt{T}$ — независимые случайные величины со стандартным нормальным распределением, а $\eta(T)=I_{1}(T) / I_{2}(T)$.

Используя явный вид решения исходной системы стохастических уравнений (см., например, [2]):

$$
\xi^{\star}(t)=e^{A t} \xi^{\star}(0)+\int_{0}^{t} e^{A(t-s)} d w^{\star}(s)
$$

где $A=\left(\begin{array}{cc}-\lambda & -\omega \\ \omega & -\lambda\end{array}\right), e^{A t}=e^{-\lambda t}\left(\begin{array}{cc}\cos \omega t & -\sin \omega t \\ \sin \omega t & \cos \omega t\end{array}\right)$, а символ $\star$ обозначает транспонирование, нетрудно показать, что

$$
\begin{aligned}
& I_{1}(T)=\xi_{1}(0) J_{1}(T)-\xi_{2}(0) J_{2}(T)+\int_{0}^{T} J_{1}(T-s) d w_{1}(s)-\int_{0}^{T} J_{2}(T-s) d w_{2}(s), \\
& I_{2}(T)=\xi_{2}(0) J_{1}(T)+\xi_{1}(0) J_{2}(T)+\int_{0}^{T} J_{2}(T-s) d w_{1}(s)-\int_{0}^{T} J_{1}(T-s) d w_{2}(s),
\end{aligned}
$$

где

$$
J_{1}(x)=\frac{\lambda+(\omega \sin \omega x-\lambda \cos \omega x) e^{-\lambda x}}{\lambda^{2}+\omega^{2}}, \quad J_{2}(x)=\frac{\omega-(\lambda \sin \omega x+\omega \cos \omega x) e^{-\lambda x}}{\lambda^{2}+\omega^{2}} .
$$

Воспользовавшись свойствами стохастических интегралов, легко установить, что $I_{1}(T)$ и $I_{2}(T)$ являются независимыми гауссовскими величинами с нулевыми математическими ожиданиями и общей дисперсией $B_{T}^{2}=b_{T}^{2}+\sigma_{T}^{2}$, где $b_{T}^{2}=\left(J_{1}^{2}(T)+J_{2}^{2}(T)\right) \sigma^{2}$, $\sigma_{T}^{2}=\int_{0}^{T}\left(J_{1}^{2}(t)+J_{2}^{2}(t)\right) d t$.

Далее, из (7) и (8) нетрудно вывести, что

$$
\begin{aligned}
& b_{T}^{2}=\frac{1}{\lambda^{2}+\omega^{2}}\left(1-2 \cos \omega T e^{-\lambda T}+e^{-2 \lambda T}\right) \sigma^{2}, \\
& \sigma_{T}^{2}=\frac{1}{\lambda^{2}+\omega^{2}}\left[T-\frac{2 \lambda}{\lambda^{2}+\omega^{2}}-\frac{2 e^{-\lambda T}}{\lambda^{2}+\omega^{2}}(\omega \sin \omega T-\lambda \cos \omega T)+\frac{1-e^{-2 \lambda T}}{2 \lambda}\right] .
\end{aligned}
$$

Отсюда следует, что при $T \rightarrow \infty$ и $\lambda<0$

$$
b_{T}^{2} \sim \frac{\sigma^{2}}{\lambda^{2}+\omega^{2}} e^{-2 \lambda T}, \quad \sigma_{T}^{2} \sim \frac{1}{2|\lambda|\left(\lambda^{2}+\omega^{2}\right)} e^{-2 \lambda T} .
$$

Покажем, что случайные величины $w_{1}^{*}$ и $I_{1}^{*}=I_{1}(T) / B_{T}$ асимптотически (при $T \rightarrow \infty)$ независимы.

Так как их совместное распределение является гауссовским, то достаточно установить их асимптотическую некоррелированность. Действительно,

$$
\begin{aligned}
\mathbf{E}\left(w_{1}^{*} I_{1}^{*}\right) & =\mathbf{E} \frac{w_{1}(T)}{\sqrt{T}} \frac{I_{1}(T)}{B_{T}}=\frac{1}{\sqrt{T} B_{T}} \mathbf{E} w_{1}(T) \int_{0}^{T} J_{1}(T-s) d w_{1}(s) \\
& =\frac{1}{\sqrt{T} B_{T}} \int_{0}^{T} J_{1}(T-s) \mathbf{E} w_{1}(T) d w_{1}(s)=\frac{1}{\sqrt{T} B_{T}} \int_{0}^{T} J_{1}(s) d s,
\end{aligned}
$$

так как $\mathbf{E} w_{1}(T) d w_{1}(s)=d s$ при $s \leqslant T$. Используя (8), находим

$$
\int_{0}^{T} J_{1}(s) d s=\frac{\lambda T}{\lambda^{2}+\omega^{2}}+\frac{\omega^{2}-\lambda^{2}-2 \lambda \omega \sin \omega T e^{-\lambda T}+\left(\lambda^{2}-\omega^{2}\right) \cos \omega T e^{-\lambda T}}{\left(\lambda^{2}+\omega^{2}\right)^{2}} .
$$

Отсюда, с учетом (9) и (10), имеем, что

$$
\lim _{T \rightarrow \infty} \mathbf{E}\left(w_{1}^{*} \cdot I_{1}^{*}\right)=0,
$$


но при этом сами случайные величины $w_{1}^{*}$ и $I_{1}^{*}$ имеют стандартное нормальное распределение. Аналогично можно доказать асимптотическую независимость $w_{1}^{*}$ от $I_{2}^{*}$, а также $w_{2}^{*}$ от $I_{1}^{*}$ и $I_{2}^{*}$.

Вспоминая (7) и учитывая сказанное выше, можно заключить, что слабый предел нормированных оценок $\lambda^{*}$ и $\omega^{*}$ имеет вид

$$
\zeta_{1}=-\left[\xi_{1} \frac{\eta}{\sqrt{1+\eta^{2}}}+\xi_{2} \frac{1}{\sqrt{1+\eta^{2}}}\right] \quad \text { и } \quad \zeta_{2}=\xi_{2} \frac{\eta}{\sqrt{1+\eta^{2}}}-\xi_{1} \frac{1}{\sqrt{1+\eta^{2}}}
$$

соответственно, где $\xi_{1}$ и $\xi_{2}$ - независимые стандартные гауссовские случайные величины, не зависящие от случайной величины $\eta$, имеющей стандартное распределение Коши.

Далее, легко показать, что $\zeta_{1}$ и $\zeta_{2}$ имеют стандартное нормальное распределение. Действительно,

$$
\begin{aligned}
\mathbf{P}\left(\zeta_{2}<x\right) & =\mathbf{P}\left(\xi_{2} \frac{\eta}{\sqrt{1+\eta^{2}}}-\xi_{1} \frac{1}{\sqrt{1+\eta^{2}}}<x\right) \\
& =\int_{-\infty}^{\infty} \mathbf{P}\left(\xi_{2} \frac{u}{\sqrt{1+u^{2}}}-\xi_{1} \frac{1}{\sqrt{1+u^{2}}}<x\right) d \mathbf{P}(\eta<u)=\mathbf{\Phi}(x),
\end{aligned}
$$

так как случайные величины $\xi=\xi_{2} u / \sqrt{1+u^{2}}-\xi_{1} / \sqrt{1+u^{2}}$ имеют стандартное нормальное распределение. Здесь $\mathbf{\Phi}(x)=(2 \pi)^{-1 / 2} \int_{-\infty}^{x} e^{-u^{2} / 2} d u$.

Теперь докажем некорреллированность $\zeta_{1}$ и $\zeta_{2}$ :

$$
\begin{aligned}
\mathbf{E}\left(\zeta_{1} \zeta_{2}\right) & =-\int_{-\infty}^{\infty} \mathbf{E}\left(\xi_{1} \frac{u}{\sqrt{1+u^{2}}}+\xi_{2} \frac{1}{\sqrt{1+u^{2}}}\right)\left(\xi_{2} \frac{u}{\sqrt{1+u^{2}}}-\xi_{1} \frac{1}{\sqrt{1+u^{2}}}\right) d \mathbf{P}(\eta<u) \\
& =-\int_{-\infty}^{\infty}\left(\frac{u}{1+u^{2}} \mathbf{E} \xi_{2}^{2}-\frac{u}{1+u^{2}} \mathbf{E} \xi_{1}^{2}\right) d \mathbf{P}(\eta<u)=0 .
\end{aligned}
$$

3 а м е ч а н и е 1 . Если $T$ (время наблюдений) является целым числом, нетрудно показать, что присутствующий в теореме нормирующий множитель $I(T) / T$ сходится п.н. к $\infty$ при $T \rightarrow \infty$.

Так как $I(T) / T=\left(I_{1}(T) / \sqrt{T}\right)^{2}+\left(I_{2}(T) / \sqrt{T}\right)^{2}$, то достаточно установить, что $I_{1}(T) / \sqrt{T} \stackrel{\text { п.н. }}{\longrightarrow} \infty$. Без ограничения общности можно считать, что $T$ является целым числом, т.е. $T=n \in \mathbf{N}$. Покажем, что имеет место сходимость $\xi_{n}=I_{1}(n) / \sqrt{n} \stackrel{\text { п.н. }}{\longrightarrow} \infty$ при $n \rightarrow \infty$, равносильная сходимости $\xi_{n}^{-1} \stackrel{\text { п.н. }}{\longrightarrow} 0$. Для этого достаточно доказать, что ряд $\sum_{n=1}^{\infty} \mathbf{P}\left(\left|\xi_{n}^{-1}\right|>\varepsilon\right)$ сходится для любого $\varepsilon>0$.

Рассмотрим

$$
P_{n}=\mathbf{P}\left(\left|\xi_{n}^{-1}\right|>\varepsilon\right)=\mathbf{P}\left(\left|\xi_{n}\right|<\frac{1}{\varepsilon}\right)=\mathbf{P}\left(\left|\frac{I_{1}(n)}{\sqrt{n}}\right|<\frac{1}{\varepsilon}\right)=\mathbf{P}\left(\left|I_{1}^{*}\right|<\frac{\sqrt{n}}{\varepsilon B_{n}}\right) .
$$

А так как $I_{1}^{*}$ имеет стандартное нормальное распределение, то отсюда получим, что

$$
P_{n} \leqslant \sqrt{\frac{2}{\pi}} \frac{\sqrt{n}}{\varepsilon B_{n}} .
$$

В силу (9) имеем

$$
B_{n}^{2} \sim\left(\frac{\sigma^{2}}{\lambda^{2}+\omega^{2}}+\frac{1}{2|\lambda|\left(\lambda^{2}+\omega^{2}\right)}\right) e^{-2 \lambda n}=c^{2}(\lambda, \omega, \sigma) e^{-2 \lambda n} .
$$

Следовательно $\sqrt{n} / B_{n} \sim \sqrt{n} e^{\lambda n} / c$ и поэтому ряд $\sum_{n=1}^{\infty} P_{n}$ сходится при $\lambda<0$.

Если $T$ не целое число, то можно рассматривать процесс $\xi(t)$ при $t \in[0,[T]]$, теряя информацию в интервале $([T], T)$, что составляет менее одной единицы времени. 
3 а м е ч а н и е 2. Для одномерной линейной диффузии

$$
d X_{t}=-\theta X_{t} d t+d w(t)
$$

асимптотическая нормальность оценки максимального правдоподобия при случайной нормировке $\left(\int_{0}^{T} X_{t}^{2} d t\right)^{1 / 2}$ в нестационарном случае $(\theta<0)$ была получена в работе [7], и там же было показано, что указанная нормировка стремится к бесконечности п.н. при $T \rightarrow \infty$.

3 а м е ч а н и е 3 . Легко понять, что основной результат работы сохраняет силу и при фиксированных начальных условиях, т.е. если $\xi(0)=\left(x_{0}, y_{0}\right)$, так как это повлияет только на предельное распределение случайной величины $\eta(T)=$ $I_{1}(T) / I_{2}(T)$, явный вид которого не играет никакой роли для асимптотической нормальности оценок.

\title{
СПИСОК ЛИТЕРАТУРЫ
}

1. Колмогоров А.Н., Арато М., Синай Я.Г. Об оценке параметров комплексного стационарного гауссовского марковского процесса. - Докл. АН СССР, 1962, т. 146, № 4, с. $747-750$.

2. Aрато М. Линейные стохастические системы с постоянными коэффициентами. М.: Наука, 1989, 304 с.

3. Липчер Р. Ш., Ширяев А. Н. Статистика случайных процессов. М.: Наука, 1974, $696 \mathrm{c.}$

4. Startsev A.N. A new approach to estimation of an autoregressive parameter. Probability Theory and Mathematical Statistics (Kiev, 1991). River Edge: World Scientific Publ., 1992, p. 377-381.

5. Стариев А.Н. Об одном новом подходе к оценке параметра сноса процесса линейной диффузии. - Узб. матем. журн., 1995, № 4, с. 72-76.

6. Durbin J. Estimation of parameters in time-series regression models. - J. Roy. Statist. Soc. Ser. B, 1960, v. 22, p. 139-153.

7. Feigin P.D. Maximum likelihood estimation for continuous-time stochastic processes. - Adv. Appl. Probab., 1976, v. 8, № 4, c. 712-736.

Поступила в редакцию

5.III.2010

Исправленный вариант

5.XII.2013

(c) 2014 г. MODARRESI N.* REZAKHAH S.*

\section{CERTAIN PERIODICALLY CORRELATED MULTI-COMPONENT LOCALLY STATIONARY PROCESSES ${ }^{1)}$}

\begin{abstract}
Определяя $X^{l s}(t)$ как случайную смесь двух стационарных процессов, где зависящие от времени случайные веса имеют экспоненциально выпуклую ковариацию, мы показываем, что этот процесс имеет многокомпонентную локально стационарную ковариационную функцию в
\end{abstract}

* Faculty of Mathematics and Computer Science, Amirkabir University of Technology, Tehran, Iran; e-mail: namomath@aut.ac.ir (Modarresi N.); rezakhah@aut.ac.ir (Rezakhah S.).

1) This work was supported by a grant of IPM (№91620034), grant of IPM (№ 91620035). 